\title{
Estrés, afrontamiento y calidad de vida relacionada a la salud en mujeres con cáncer de mama
}

\author{
Stress, Coping And Health-Related \\ Quality Of Life In Breast Cancer Women
}

\author{
Vanessa Lostaunau ${ }^{1}$ \\ Claudia Torrejón ${ }^{2}$ \\ Mónica Cassaretto ${ }^{3}$ \\ Pontificia Universidad Católica del Perú, Perú
}

Resumen. Este estudio examina la contribución que tienen el estrés traumático, el estrés percibido y el afrontamiento sobre los componentes físico y mental de la CVRS de 53 mujeres diagnosticadas con cáncer de mama. Los instrumentos utilizados fueron el cuestionario de salud SF-36, el inventario de estimación del afrontamiento COPE, la escala de estrés percibido EEP y la adaptación peruana de la escala de estrés traumático. Se encontraron niveles promedio y altos de CVRS. La condición de migración, el tipo de tratamiento y el estrés traumático explican el 30\% de la variabilidad del componente físico; mientras que, el estrés traumático, el estrés percibido y el estilo orientado a la emoción, explican el $43 \%$ de la variabilidad del componente mental.

Palabras clave. Calidad de vida relacionada a la salud (CVRS), afrontamiento, estrés percibido, estrés traumático, cáncer de mama, migración y tipo de tratamiento.

Abstract. This study analyzes the contribution that traumatic stress, perceived stress, and coping have on the variability of physical and metal components of the HRQoL of 53 women with breast cancer. The instruments used include the SF36 health survey, the COPE Inventory, the Perceived Stress Scale PSS and the Peruvian adaptation of the traumatic stress scale. The analysis found medium and high levels of global HRQoL. The migration status, type of treatment and traumatic stress account for $30 \%$ of the variability of the physical component of HRQoL, whereas traumatic stress, perceived stress and emotional oriented style account the $43 \%$ of the variability of the mental component.

Keywords. Health-related quality of life (HRQoL), coping, perceived stress, traumatic stress, breast cancer, migration and type of treatment.

\footnotetext{
${ }^{1}$ Vanessa Lostaunau. Pontificia Universidad Católica del Perú, Perú. E-mail: v.lostaunau@pucp.edu.pe

${ }^{2}$ Claudia Torrejón. Pontificia Universidad Católica del Perú, Perú. E-mail: claudia.torrejon@pucp.pe

${ }^{3}$ Mónica Cassaretto. Pontificia Universidad Católica del Perú, Perú. Dirección postal: Av. Universitaria 1801, San Miguel, Lima 32, Perú

511-6262000 anexo 4598., Perú. E-mail: mcassar@pucp.edu.pe
}

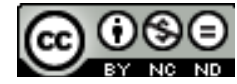




\section{Introducción}

El cáncer es una enfermedad temida en todas las culturas. El diagnóstico de esta genera una asociación inmediata al sufrimiento, al dolor, a la muerte (Die Trill, 2003) y podría producir grandes repercusiones en la calidad de vida de quienes lo padecen (Atef-vahid, et al., 2011). A nivel mundial, el cáncer se encuentra entre las principales causas de morbilidad y mortalidad y, particularmente el cáncer de mama, representa el 16\% de todos los cánceres femeninos (Organización Mundial de la Salud [OMS], 2012).

Diversos estudios han indicado que la población de mujeres con cáncer de mama es vulnerable a desarrollar altos niveles de morbilidad psicológica. Algunos reportan la presencia de estados depresivos, altos niveles de ansiedad (Karakajun, Ilknur, Sahin, Orcin, Alanyali, \& Kinay, 2010; Koch, 2007; Stafford, Judd, Gibson, Komiti, Mann, \& Quinn, 2013), sensación de desesperanza (Van Laarhoven, Schilderman, Bleijenberg, Donders, Vissers, Verhagen, \& Prins, 2011), así como malestar general y dolores físicos (Avis, Crawford, \& Manuel, 2005; Galloway, Baker, Giglio, ... \& Borckardt, 2012).

Para algunos autores, el malestar emocional está, en gran medida, originado por los efectos específicos del diagnóstico y su tratamiento (Rincón, Pérez, Borda, \& Martín, 2010). Para otros, la presencia de emociones negativas está asociada a la variedad de estresores físicos, mentales, sociales y laborales a los que deben hacer frente este tipo de pacientes (Da Silva \& Dos Santos, 2010; Yang, Brothers, \& Andersen, 2008). Todos estos investigadores coinciden en que se trata de una enfermedad que tiende a comprometer negativamente la calidad de vida.

Según la OMS (1995), la calidad de vida hace referencia a la percepción subjetiva y temporal que tienen las personas sobre su estado de bienestar físico, psíquico, social y espiritual, en asociación con su contexto histórico, cultural y social. También, influyen su sistema de valores y creencias y sus metas, expectativas y estándares.
En individuos con enfermedades crónicas, se encuentra un quiebre importante en una de las dimensiones que conforman la calidad de vida: la pérdida de la salud. Es por ello que se prefiere usar el término calidad de vida relacionada con la salud (siglas CVRS), como una noción más restringida de la calidad de vida. En pacientes oncológicos, este concepto alude al impacto que tienen la enfermedad y su consecuente tratamiento (secuelas y efectos secundarios) sobre cómo dimensionan su bienestar y su vida en general, en función de la percepción de limitaciones físicas, psicológicas, sociales y de disminución de oportunidades (Schwartzmann, 2003).

A pesar de la elevada incidencia de morbilidad psicológica, diversos estudios señalan que la CVRS se encuentra relativamente preservada, tanto en términos generales como a nivel de sus componentes físico y mental, cuando se sufre cáncer de mama (Kershaw, Northouse, Kritpracha, Schafenacker, \& Mood, 2004; Laos, 2010; Ransom, Jacobsen, Schmidt, \& Andrykowski, 2005; Torres, 2011). Además, muchos estudios resaltan que su impacto puede ser modesto e incluso transitorio en la mayoría de los casos (Kershaw et al., 2004; Ransom et al., 2005); sin embargo, pasados varios meses de tratamiento, existe cierta capacidad de recuperación en las pacientes (Costa-Requena, Rodríguez, \& Fernández-Ortega, 2012; Golden-Kreutz, Thornton, Wells-DiGregorio, ... \& Andersen, 2005). A pesar de esto, existe un grupo pequeño de mujeres que sí demuestra un deterioro más significativo en la CVRS y son ellas las vulnerables a ver afectadas sus vidas de forma significativa (Gaviria, Vinaccia, Riveros, \& Quinceno, 2007; Kershaw, Northouse, Kritpracha, Schafenacker, \& Mood, 2004).

Aparentemente, existen ciertos factores, sociodemográficos y propios de la enfermedad, que se vincularían con el impacto que esta última genera. Entre ellos, se señala el lugar de procedencia al momento de ir a un centro de atención y tratamiento de enfermedades oncológicas (Laos, 2010), el nivel educativo (Reich \& Remor, 2014) y la ocupación actual de la paciente (Torres, 2011). Otras condiciones serían 
la posibilidad de muerte inminente, la presencia de comorbilidad y el dolor crónico (Gaviria et al. 2007; Reich \& Remor, 2014).

Sin embargo, existe menor acuerdo respecto a otras variables, como el tipo de tratamiento recibido por la paciente: quimioterapia, radioterapia u hormonoterapia (Laos, 2010; Torres, 2011). También, influyen el tipo de cirugía y el tiempo que se está recibiendo el tratamiento (Filazoglu \& Griva, 2008; Laos, 2010).

Por otro lado, existe evidencia de que una condición relevante a la forma en que se sobrelleva una enfermedad se relacionaría con las condiciones de vida de las pacientes. Entre estas, sobresale la experiencia de migrar de su zona de residencia (Laos, 2010; Torres, 2011). En el Perú, las migraciones internas responden, principalmente, a las desigualdades económicas y sociales presentes entre la forma de vida de las provincias y la capital (Granel, 2008; Yamada, 2010). En lo que respecta al sistema sanitario del país, existen solo cinco institutos regionales para el tratamiento del cáncer. El principal y más completo es el de Lima (Ministerio de Salud [MINSA], 2012), por lo que cerca del 50\% de los pacientes oncológicos que se atienden en el principal centro de atención oncológico de la capital son migrantes (Instituto Nacional de Enfermedades Neoplásicas [INEN], 2016).

Los pacientes migrantes deben enfrentarse a la necesidad de dejar sus culturas de origen para acomodarse en un contexto nuevo y poco familiar (Ferrer, Palacio, Hoyos \& Madariaga, 2014), lo que supone no solo lidiar con la pérdida de la salud sino, también, una importante disminución en su soporte social, vivenciar experiencias de discriminación y carecer de suficiente información sobre los servicios de salud (Gil, Wagner, \& Vega, 2000; Shishehgar, Gholizadeh, DiGiacomo, \& Davidson, 2015).

Además, suele ocurrir una separación transitoria de sus costumbres, actividades cotidianas y, en ocasiones, la lengua. Todo esto llevaría a que los pacientes tengan que sobrellevar múltiples duelos (Granel, 2008). Adicionalmente, estos pacientes suelen enfrentarse a otros estresores importantes como son los económicos y fi- nancieros pues, si bien hay programas nacionales que subvencionan los gastos de traslado del paciente, estos no cubren la estadía del paciente ni del familiar que lo suele acompañar, por lo que suele haber una reducción del ingreso económico familiar (MINSA, 2012).

Por todos estos motivos, Hernández, Lorenzo y Nacif (2009) consideran que la migración es un factor de riesgo para la salud debido a la alta vulnerabilidad biopsicosocial a la que el paciente se enfrentaría. Este es un aspecto alarmante que se relaciona con la evidencia de que la población minoritaria o inmigrante oncológica, puede tener menor supervivencia en comparación con la de pacientes locales o no migrantes (New Jersey Department Health, 2013; Hernández et al., 2009).

Las investigaciones psicológicas encuentran que uno de los factores más vinculado a la CVRS sería el afrontamiento. Este es considerado como un estabilizador que ayuda a los individuos a mantener la adaptación psicosocial durante períodos de estrés, tales como los que son disparados por la enfermedad y el gran número de cambios de vida significativos que este implica (Avis, et al., 2005; Filazoglu \& Griva, 2008; Kershaw et al., 2004; Ransom et al., 2005; Tarakeshwar, et al., 2006; Yang et al., 2008).

El afrontamiento se define como los esfuerzos cognitivos y conductuales realizados para manejar situaciones que han sido evaluadas como potencialmente amenazantes o estresantes. Su función es eliminar o reducir el distrés emocional asociado a las condiciones estresantes que experimenta un individuo (Lazarus \& Folkman, 1986); por ello, es contemplado también como un regulador de emociones (Lazarus \& Lazarus, 2000).

Se han encontrado importantes relaciones entre la CVRS y los tipos de afrontamiento en pacientes con cáncer de mama. En esa línea, se reporta una relación directa entre el componente mental de la CVRS y el uso de la estrategia afrontamiento activo (Constanzo et al., 2006; Kershaw et al., 2004; Van Laarhoven et al., 2011). Además, se hallan relaciones inversas entre CVRS con el afrontamiento evitativo y estrategias como el uso desentendimiento o la focalización y liberación de las emociones (Constanzo et al., 2006; 
Kershaw et al., 2004; Van Laarhoven et al., 2011). Asimismo, se encuentra que mayores niveles de CVRS se relacionan con un mayor uso de las estrategias aceptación y religiosidad (Van Laarhoven et al., 2011).

Cabe señalar que la mayoría de estos estudios asumen a la enfermedad oncológica como estresante en sí misma, sin diferenciar el nivel de estrés percibido y el impacto traumático que esta puede ocasionar. Para algunos autores, la experiencia del cáncer puede estar marcada por altos niveles de estrés y disrupción del proceso de ajuste a la enfermedad (Constanzo et al., 2007). En general, se encuentra que el estrés está asociado con preocupaciones acerca del cáncer, incluyendo problemas físicos y temor a la recurrencia de la enfermedad, y tiende a prevalecer a pesar de haber sobrevivido a la enfermedad (Constanzo et al., 2007, Golden-Kreutz et al., 2005).

Se conoce que el nivel de estrés percibido se relaciona de manera inversa con la CVRS (Costa-Requena et al., 2012; Golden-Kreutz et al., 2005); sin embargo, esta relación no es lineal ni simple. Costa-Requena et al. (2012), estudiaron dichas variables en 67 pacientes con cáncer de mama, y encontraron que el estrés percibido se relacionaba de forma distinta con la CVRS según el periodo de tiempo en que este es evaluado: son más significativos durante los periodos de pre-tratamiento y tratamiento.

Un aspecto clave para entender la dinámica entre el estrés percibido, el afrontamiento y la CVRS, es suponer que el cáncer no solo puede ser considerado como un evento de vida potencialmente estresante para las personas, sino que este puede activar la presencia de respuestas similares a las que ocurren en presencia de un trastorno de estrés post-traumático (TEPT) o, en su defecto, disparar síntomas sub-clínicos de este último (Koch, 2007; Koopman et al., 2002; O’Connor, Christensen, Jensen, Moller, \& Zachariae, 2011).

Si bien la relación entre dicho diagnóstico y el cáncer aún es poco clara, la revisión bibliográfica sugiere que la prevalencia del TEPT en pacientes oncológicos recién diagnosticados oscila entre 3\% y 14\% (Gurevich, Devins, \& Rodin, 2002; Shelby, Golden-Kreutz, \& An- dersen, 2008). También, se evidencia que puede aumentar al 35\% en pacientes evaluados luego de finalizar el tratamiento, reducirse a $18.5 \%$ en pacientes en un periodo post-operatorio (Koch, 2007) o ser más frecuente en pacientes con tratamiento adyuvante más agresivo y con mayor progreso de la enfermedad (Gurevich et al., 2002). Otros estudios han reportado prevalencia de altos niveles de estrés en pacientes con cáncer de mama, los cuales, si bien no cumplen con los criterios para el TEPT, se acercan a la descripción del este por la presencia de síntomas tales como pensamientos intrusivos recurrentes, evitación cognitiva y conductual de los eventos vinculados a la enfermedad, adormecimiento e hiperactivación (Golden-Kreutz et al., 2005).

Pocas investigaciones estudian la forma en que la CVRS interactúa con el estrés percibido, el estrés traumático y el afrontamiento. Una de ellas fue la llevada a cabo por Golden-Kreutz et al. (2005), quienes evaluaron a 112 pacientes con este tipo de cáncer en tres momentos distintos (desde el diagnóstico hasta el seguimiento); encontraron que los niveles altos de estrés, tanto percibido como traumático, detectados al inicio del tratamiento, pueden predecir menores niveles de CVRS a lo largo del tiempo. Además, los síntomas de estrés traumático al inicio de la evaluación fueron los predictores más poderosos de la CVRS en los tres momentos, con un mayor impacto en el componente mental.

Por otro lado, Yang et al. (2008), quienes evaluaron a 65 pacientes con cáncer de mama, encontraron que el estrés percibido resulta ser un factor determinante en la relación entre afrontamiento y la CVRS, pero esto ocurre solo cuando los pacientes reportaron síntomas de estrés intensos. Es ahí donde el afrontamiento activo correlacionó positivamente con la CVRS; sin embargo, cuando presentaron estrés traumático, se anuló dicha correlación. Finalmente, se encontró que a mayor presencia de síntomas de estrés y de indicadores de estrés traumático, se hacía más probable el uso de estilos evasivos de afrontamiento.

Se observa que el diagnóstico y el tratamiento de cáncer de mama son eventos que pueden generar altos niveles de estrés (Golden-Kreutz et al., 2005; Koch, 2007; Yang et al., 2008) e incluso delinear la presencia de sintomatología 
similar a la de un TEPT (Koopman et al., 2002) capaces de afectar la CVRS en las pacientes con cáncer de mama. A pesar de eso, aún falta examinar cuáles son los elementos que explican la variabilidad en la CVRS.

El presente estudio busca examinar el poder explicativo que tienen algunas variables sociodemográficas, de enfermedad, estrés percibido, estrés traumático y los tipos de afrontamiento, sobre los componentes físico y mental de la CVRS de un grupo de pacientes con cáncer de mama que asisten a recibir tratamiento a un centro de atención especializada en cáncer en el Perú. Se espera que la presencia de niveles altos de estrés percibido, estrés traumático, afrontamientos evitativos y la presencia de condición de migración y tratamientos más agresivos sean los que impacten de manera más negativa en los distintos componentes de la CVRS de las pacientes evaluadas.

\section{Método}

\section{Participantes}

El tipo de muestreo fue intencional (Hernández, Fernández \& Baptista, 2010) e incluyó a aquellas pacientes mujeres adultas que han sido diagnosticadas por primera vez con cáncer de mama en un periodo entre 3 a 12 meses y que se asisten a un centro nacional de atención de enfermedades neoplásicas en la ciudad de Lima.

La muestra está compuesta por 53 mujeres adultas, cuyo rango de edad fluctúa entre 30 y 69 años $(M=$ 48.06, $D T=8.84)$ La mayoría tiene un tiempo de diagnóstico de 3 a 6 meses $(57 \%$ ) y presentan estadios clínicos I (11.3\%), II (62.3\%) y III (26.4\%). Todas las mujeres asisten a un centro especializado en enfermedades neoplásicas en la ciudad de Lima y se han sometido a una extirpación total $(91 \%)$ o parcial $(9 \%)$ de la mama. La mayoría se encuentra en un periodo post-operatorio (77.4\%); es decir, en el área de hospitalización, mientras que otras $(22.6 \%)$ acuden de manera ambulatoria.

Se excluyó a las pacientes que tenían presencia de metástasis o estadio IV, comorbilidad con alguna enfermedad grave o condición psiquiátrica, historia previa de cáncer de mama, deterioro cognitivo, analfabetismo y un nivel educativo básico de solo primaria.

Todas las mujeres aceptaron participar de manera voluntaria y, para ello, firmaron un consentimiento informado. La administración fue individual, a modo de entrevista y, dada la movilización en el tema y de acuerdo a los lineamientos éticos, se ofreció un espacio de consejería para cerrar esta experiencia. El proyecto de investigación que fundamentó el presente estudio fue evaluado y aprobado en su momento por el comité de ética e investigación del centro de atención.

\section{Instrumentos}

La CVRS fue medida a través de la adaptación española del Cuestionario de salud SF-36 versión 1 (Alonso, Prieto \& Antó, 1995), el cual fue desarrollado originalmente por Ware y Sherbourne (1992) con el objetivo de crear una escala genérica que proporcione un perfil del estado de salud de las personas. Es uno de los instrumentos con mayor potencial (Alonso et al., 1995) y utilidad para evaluar la CVRS, pues permite comparar el impacto que producen las distintas enfermedades y sus tratamientos y valorar el estado de salud físico y psicológico de los pacientes (Vilagut et al., 2005).

El SF-36 consta de 36 ítems que describen distintos indicadores de salud; entre ellos se encuentran la medida de salud percibida global, dos indicadores generales de salud (física y mental) y ocho áreas específicas. Asimismo, ha sido traducido a distintos idiomas como parte del proyecto "Internacional Quality of Life Assessment" (IQOLA); por ello, el instrumento ha sido adaptado en más de 40 países y existen más de 500 publicaciones con las versiones traducidas del inglés.

Respecto a sus propiedades psicométricas, existen estudios llevados a cabo por el proyecto IQOLA que encuentran que la confiabilidad de la prueba excede el .80 para las ocho dimensiones y el .90 para los indicadores generales de salud. En estos estudios se confirma, además, la estructura factorial del instrumento con varianzas explicadas que superan el 76\% (Ware, 1998). En el Perú, Salazar y Bernabé (2012) han evaluado las propiedades psicométricas del 
instrumento en una muestra de 4344 personas entre 15 y 64 años. Los índices de consistencia interna alfa de Cronbach para las ocho dimensiones específicas se ubican entre .66 y .92. El análisis factorial confirmatorio mostró que un modelo de 8 factores de primer orden y 2 de segundo orden era la estructura factorial más adecuada, ya que mostraba mejores índices de ajuste a los datos de $C F I=.96$ y $\operatorname{RMSE} A=$ .05 en comparación con modelos alternativos.

De manera similar, en el presente estudio, se obtuvieron puntuaciones alfa de Cronbach que fluctuaron entre .62 y .88 para todas las dimensiones de la prueba, mientras que, a nivel de los componentes físico y mental, se obtuvieron puntuaciones de $.81 \mathrm{y}$ .83 , respectivamente.

El afrontamiento fue medido a través del Inventario de Estimación de Afrontamiento COPE, el cual fue creado por Carver, Scheier y Weintraub (1989) con el objetivo de conocer las maneras en que las personas responden a las situaciones estresantes. Está compuesto por 60 ítems organizados en 15 sub-escalas, estas se responden mediante un formato Likert de 4 escalas de frecuencia.

La versión original del COPE (53 ítems) fue aplicada a 978 sujetos. En términos de consistencia interna, las escalas de la prueba presentaron índices alfa de Cronbach entre .45 hasta .92 y las mediciones test-retest dieron rangos entre .46 y .86 a las 8 semanas. La validez de constructo fue calculada mediante un análisis factorial de rotación oblicua, obteniendo 12 factores con eigenvalues mayores a 1.0, de los cuales 11 fueron fácilmente interpretados y los otros no llegaron a una carga factorial de .30 (Carver et al., 1989).

La investigación realizada por Cassaretto y Chau (2016) en 300 estudiantes universitarios utiliza la versión del COPE 60 traducida al español y señala puntajes alfa de Cronbach que oscilan entre .54 y .91. Para la validez, en principio se hizo un análisis factorial exploratorio con rotación oblicua (KMO $=.80, p<0.001)$ del que emergen 15 factores que explican una varianza de $65 \%$ de la prueba. Luego, se realizó un análisis factorial exploratorio de segundo orden, el cual arrojó una estructura de 3 factores que explicaban el $55.92 \%$ de la varianza $(K M O=$ $.69, p<.01)$. Finalmente, se evaluaron 3 modelos de organización de las escalas del COPE. Los resultados obtenidos con análisis factorial confirmatorio sugieren un uso prudencial del modelo 3 factores que está compuesto por los estilos: orientado a lo social/ emocional, a la evitación, y a la tarea/aproximación (Cassaretto \& Chau, 2016).

En el presente estudio, se encontró que la estructura de 3 estilos era la pertinente y se encontraron puntajes alfa de Cronbach de .86, .81 y .75 para los mismos (orientado al problema, orientado a la emoción y evitativo, respectivamente). Las puntuaciones para las estrategias fluctuaron entre .56 y .85 , aunque las escalas afrontamiento religioso y uso de alcohol/ drogas presentaron indicadores bajos $(.23$ y .00 , respectivamente). Estas dos últimas se justifican debido a que casi la totalidad de participantes respondieron que usan con mucha frecuencia la religión, mientras que casi nunca usan alcohol o drogas como forma de afrontamiento al estrés. Comprendiendo ambas estrategias en el contexto de una enfermedad, se mantuvieron dichas escalas.

La evaluación del estrés percibido fue realizada a través de la Escala de estrés percibido EEP, la cual fue elaborada por Cohen, Kamarck y Mermelstein en 1983. Esta prueba busca conocer qué tan estresantes perciben las personas los eventos de la vida cotidiana durante el último mes. Se utilizó la versión traducida y validada al español por Remor y Carrobles (2001). Dicha escala de auto-informe consta de 14 ítems, los cuales se agrupan en dos factores de 7 ítems cada uno. El primer factor denominado "Expresión de Estrés" evalúa la percepción de pérdida de control en el manejo del estrés; mientras que, el segundo factor, Control del Estrés, hace referencia a la percepción de satisfacción o éxito en el manejo del estrés. La escala utiliza un formato de respuesta tipo Likert de cinco alternativas con un rango de 0 (Nunca) a 4 (Siempre).

En la creación de la prueba original Cohen, Kamarck y Mermelstein (1983) aplicaron la escala a tres muestras diferentes y obtuvieron una consistencia 
interna mediante el alfa de Cronbach de .84, .85 y .86, respectivamente, para cada muestra. La confiabilidad test-retest a los 2 días y 6 semanas fue .85 para la primera y .55 para la tercera muestra. Asimismo, la validez concurrente se evidenció por medio de las correlaciones significativas entre la PSS y el Life-Event Scores (Holmes y Rahe, 1976), donde se obtuvieron correlaciones de $.20, .17$ y .39 , respectivamente, para cada muestra. En el 2006, Remor estudió las propiedades psicométricas del instrumento con una muestra de 440 españoles. La consistencia interna obtenida a través del alfa de Cronbach, arrojó un puntaje de .81 la confiabilidad test-retest, a las 2 semanas, fue de .73. La validez concurrente quedó evidenciada a través de las correlaciones significativas (.71 y .64) entre el EEP y el Hospital Anxiety and Depression Scale (HADS) que miden constructos similares (ansiedad y distrés). Dichos resultados coinciden con los niveles de confiabilidad hallados en el presente estudio, los cuales, mediante el estadístico alfa de Cronbach, alcanzaron puntajes globales de .80, de .72 para el factor 1, expresión de estrés y .76 para el factor 2, control de estrés.

Finalmente, el impacto del estrés traumático fue medido mediante la versión peruana de la Escala de impacto de los eventos IES-R creado por Horowitz, Wilner y Álvarez (1979), traducida y adaptada en el Perú por Gargurevich (2006). El objetivo de la prueba radica en explorar el impacto psicológico que tienen determinados eventos traumáticos sobre las personas (Sundin \& Horowitz, 2002). El IES-R contiene 22 ítems y está organizado en cuatro dimensiones: intrusión (aparición de pensamientos o imágenes relacionados al evento traumático); evitación (evitación de pensamientos, sentimientos y situaciones relacionadas con el evento estresor); hiperactivación (activación fisiológica manifestada en síntomas físicos); y una última escala agregada por Gargurevich (2006) llamada desorden del sueño (dificultad para conciliar o mantener el sueño). El instrumento en mención se puntúa en una escala tipo Likert de 5 puntos, cuyas instrucciones se enfocan en el grado de estrés asociado con cada síntoma.
En su investigación, Gargurevich (2006) obtuvo coeficientes alfa de Cronbach para cada área, entre .78 a .94 y estudió la validez convergente a través de un análisis correlacional con un inventario equivalente (Clinician Administered PTSD Scales CAPS; Blake et al., 1995). De este modo, encontró altas correlaciones entre las subescalas equivalentes ( $r$ de Pearson de .24 a .39).

Los resultados obtenidos en la presente investigación arrojan un alfa de Cronbach para la prueba de .85 . Además, los puntajes de sus áreas obtuvieron .73 (intrusión), .68 (evitación), .61 (hiperactivación) y .85 (desorden del sueño).

\section{Procedimiento}

La administración de las pruebas se realizó en un centro especializado en enfermedades neoplásicas de la ciudad de Lima, luego de coordinar y conseguir la autorización respectiva. La evaluación fue realizada a modo de entrevista, a un único grupo de pacientes y en un solo momento en el tiempo. Finalizada la evaluación, se brindó un espacio de consejería a cada participante.

\section{Estrategias de análisis}

Los datos se analizaron con el programa estadístico SPSS versión 17.0. Inicialmente, se identificó la consistencia interna de cada instrumento mediante el coeficiente alfa de Cronbach, así como la correlación ítem-test. Posteriormente, los resultados fueron analizados inicialmente con estadísticos descriptivos y de frecuencia; se realizó, además, la prueba de normalidad de Kolmogorov-Smirnov, la cual indicó que los puntajes en la mayoría de dimensiones de ambas pruebas seguían una distribución normal.

No obstante, según las estadísticas de asimetría y curtosis de Kline (2010), no existiría una falta severa de normalidad en ninguna de las dimensiones. Tomando en cuenta ello, se realizaron contrastes de medias T-Student y para las correlaciones, se utilizó el estadístico $r$ de Pearson. Posteriormente, se realizaron los análisis de regresión jerárquica para la CVRS Física y para la CVRS Mental incluyendo las variables psicológicas, sociodemográficas y de enfermedad que 
estuvieron significativamente correlacionadas con la CVRS, tal como se realizó en el estudio de GoldenKreutz et al. (2005). Estos análisis implicaron hacer modelos separados cuando se encontraron asociaciones significativas con cada componente a nivel de estilos y de estrategias de afrontamiento. Previamente al modelo de regresión, se realizó un análisis de covarianzas para conocer el efecto atribuible que tiene cada variable de estudio sobre la CVRS.

\section{Resultados}

A continuación, se procederá a presentar los resultados obtenidos para responder a los objetivos de la investigación. Los análisis descriptivos de la CVRS detallados en la tabla 1, refieren que el componente físico obtuvo puntajes dentro del quintil promedio; mientras que el componente emocional arroja puntajes que se ubicó en el quintil alto. Con relación a las dimensiones, la mediana más alta la obtuvo el rol emocional, ubicándose en el quintil muy alto, y la más baja el rol físico, el cual se ubica en el quintil bajo.

$\mathrm{Al}$ analizar si la CVRS obtenía variaciones según las variables sociodemográficas, se encontraron diferencias significativas en el componente físico con las variables migración y tipo de tratamiento. Se encuentra que las pacientes no migrantes (provenientes de Lima) obtienen puntajes más elevados que las pacientes migrantes en dicho componente (Mnomigrante $=63.49$, Mmigrante $=52.82, t=-2.48, p=.16, d=.68)$. En relación con el tipo de tratamiento, los análisis revelaron diferencias estadísticamente significativas entre las pacientes que recibieron tratamiento quimioterapéutico y las pacientes que no recibieron ningún tipo de tratamiento; las primeras reportaron mejores puntuaciones en el componente físico en comparación con las mujeres que no recibieron tratamiento alguno (Mquimioterapia = 63.70, Mninguno $=53.21, t=2.316, p=.02, d=.69)$.

Con relación al afrontamiento se presentan, en la tabla 2, los descriptivos de esta variables. Se puede observar que el estilo más utilizado por las pacientes corresponde al orientado a la emoción, mientras que el menos utilizado fue el afrontamiento por evitación. A nivel de las estrategias, se obtuvo un mayor uso de acudir a la religión, seguido de la reinterpretación positiva y crecimiento, así como un menor despliegue de uso de sustancias y desentendimiento conductual.

En la tabla 3, pueden encontrarse los análisis de correlación entre la CVRS y sus componentes con las variables psicológicas del estudio. Se presentan aquellas que fueron estadísticamente significativas; se hallaron relaciones inversas entre las variables afrontamiento, estrés percibido y estrés traumático con los componentes de la

Tabla 1

Descripción de los componentes y las dimensiones de la CVRS

\begin{tabular}{lcccc}
\hline & $M$ & $D T$ & \multicolumn{2}{c}{ IC 95\% } \\
\hline Función física & 60.00 & 24.29 & 53.32 & 66.68 \\
Rol físico & 33.96 & 35.73 & 24.12 & 43.81 \\
Dolor corporal & 69.95 & 23.42 & 63.50 & 76.41 \\
Salud general & 66.70 & 18.71 & 61.54 & 71.85 \\
Vitalidad & 69.25 & 21.67 & 63.27 & 75.22 \\
Función social & 68.63 & 22.68 & 62.38 & 74.88 \\
Rol emocional & 65.41 & 39.75 & 54.45 & 76.36 \\
Salud mental & 67.32 & 19.03 & 62.07 & 72.57 \\
\hline Componente físico & 57.65 & 16.29 & 53.16 & 62.14 \\
Componente mental & 67.65 & 19.41 & 62.30 & 73.00 \\
\hline N=53 & & & &
\end{tabular}


Tabla 2

Descripción de estilos y estrategias de afrontamiento

\begin{tabular}{|c|c|c|c|c|}
\hline \multirow{2}{*}{ Estilos de Afrontamiento } & \multirow[t]{2}{*}{$M$} & \multirow[t]{2}{*}{$D T$} & \multicolumn{2}{|c|}{ IC 95\% } \\
\hline & & & & \\
\hline Orientado al problema & 56.38 & 10.93 & 53.36 & 59.39 \\
\hline Orientado a la emoción & 60.92 & 8.90 & 58.46 & 63.37 \\
\hline Orientado a la evitación & 39.32 & 8.16 & 37.07 & 41.57 \\
\hline \multicolumn{5}{|l|}{ Estrategias de Afrontamiento } \\
\hline Afrontamiento activo & 11.74 & 2.40 & 11.07 & 12.40 \\
\hline Planificación & 12.06 & 2.87 & 11.27 & 12.85 \\
\hline Supresión de actividades competentes & 10.70 & 2.81 & 9.92 & 11.47 \\
\hline Postergación del afrontamiento & 10.53 & 2.63 & 9.80 & 11.25 \\
\hline Soporte social instrumental & 11.36 & 3.39 & 10.42 & 12.29 \\
\hline Soporte social emocional & 11.10 & 3.22 & 10.22 & 11.99 \\
\hline Reinterpretación positiva & 13.21 & 2.44 & 12.53 & 13.88 \\
\hline Aceptación & 12.62 & 2.60 & 11.91 & 13.34 \\
\hline Acudir a la religión & 14.64 & 1.51 & 14.23 & 15.06 \\
\hline Enfocar y liberar emociones & 9.35 & 3.18 & 8.46 & 10.22 \\
\hline Negación & 8.83 & 2.96 & 8.01 & 9.65 \\
\hline Humor & 9.13 & 3.89 & 8.06 & 10.21 \\
\hline Desentendimiento mental & 10.64 & 2.65 & 9.91 & 11.37 \\
\hline Desentendimiento conductual & 6.70 & 2.99 & 5.87 & 7.52 \\
\hline Uso de sustancias & 4.02 & 0.14 & 3.98 & 4.06 \\
\hline
\end{tabular}

CVRS. Estos hallazgos indican relaciones significativas y de magnitud media acercándose a alto (siguiendo el criterio de Cohen), por lo que se sustenta que, a mayor nivel de alguna de las variables independientes de estudio, menor será el nivel del componente con el que se encuentra relacionado y viceversa.

Cumpliendo con el objetivo principal del estudio, se realizaron análisis de regresión jerárquica para cada componente de la CVRS. En la tabla 4 se presentan los resultados de la regresión jerárquica para la CVRS Física. Se tomaron como variables independientes aquellas que fueron significativas en los análisis previos. Ingresan, primeramente, las características de sociodemográficas y de enfermedad. En seguida, en un segundo tipo de estrés, la estrategia de afrontamiento que haya sido significativa; no hubo necesidad de presentar un segundo modelo que incorporara los estilos de afrontamiento, ya que ninguno de ellos se relacionó con la CVRS Física. En lo que respecta al componente físico, se observa que la migración, el tipo de tratamiento y el estrés traumático consiguieron explicar el 30\% de su variabilidad, con una $\mathrm{F}_{(52)}=6.976$, $p<.001$. En este caso, el afrontamiento no consiguió explicar la variabilidad de dicho componente.

Se observan, en la tabla 5, los análisis de regresión jerárquica que toma la CVRS Mental como variable dependiente y como variables independientes a los dos tipos de respuesta de estrés y el afrontamiento. Debido a la posible redundancia entre estilos y estrategias, se hicieron modelos separados; en uno de ellos, se incor- 
Tabla 3

Relación del afrontamiento, el estrés percibido y el estrés traumático con los componentes de la CVRS

\begin{tabular}{lcc}
\hline & \multicolumn{2}{c}{ CVRS } \\
\cline { 2 - 2 } Afrontamiento & Componente físico & Componente mental \\
\cline { 2 - 2 } Supresión de actividades competentes & $-.35^{* *}$ & \\
Enfocar y liberar emociones & $-.35^{*}$ \\
Soporte social emocional & $-.29^{*}$ \\
Estilo orientado a la emoción & $-.29^{*}$ \\
Estrés percibido & $-.46^{* *}$ \\
Estrés traumático & $-.45^{* *}$ & $-.54^{* *}$ \\
\hline
\end{tabular}

$N=53,{ }^{*} p<.05,{ }^{* *} p<.01$

Tabla 4

Análisis de regresión como predictor de la CV RS física

\begin{tabular}{|c|c|c|c|c|}
\hline \multirow[b]{2}{*}{ Pasos y predictores } & \multicolumn{2}{|c|}{ Estadística por pasos } & \multicolumn{2}{|c|}{ Estadística por predictor } \\
\hline & $\mathrm{R}^{2}$ & $\Delta R^{2}$ & $\beta$ & $t$ \\
\hline & \multicolumn{4}{|c|}{ Ingreso de variables } \\
\hline \multicolumn{5}{|l|}{ Paso 1} \\
\hline Migración & .11 & $.11 * *$ & .24 & 1.93 \\
\hline Tipo de tratamiento & .18 & $.07 * *$ & -.18 & -1.41 \\
\hline \multicolumn{5}{|l|}{ Paso 2} \\
\hline Estrés traumático & .30 & $.12^{* * *}$ & -.30 & $-2.23 * *$ \\
\hline \multicolumn{5}{|l|}{ Paso 3} \\
\hline Supresión de actividades competentes & .32 & .02 & -.16 & -1.20 \\
\hline
\end{tabular}

poran solo las estrategias de afrontamiento que correlacionaron con la CVRS Mental; en otro modelo, el estilo de afrontamiento que se relacionó de la misma manera. Los análisis encuentran que el estrés traumático, el estrés percibido y las estrategias Búsqueda de soporte social emocional y enfocar y liberar emociones explican el 37\% de su variabilidad, aunque las estrategias de afrontamiento no fueron significativas dentro del modelo. Por otro lado, los análisis que incorporan ambos tipos de estrés y el estilo de afrontamiento orientado a la emoción consiguieron explicar el 43\% de la variabilidad de la CVRS.

\section{Discusión}

En general, se encuentra que la evaluación de la CVRS es sumamente relevante para conocer niveles de satisfacción física, emocional, social; así como el funcionamiento cotidiano de las pacientes con cáncer de mama. A grandes rasgos, se observa que la valoración que hacen las participantes de su CVRS en los dos componentes es favorable, lo que coincide con los hallazgos obtenidos en el Perú en los estudios de Torres (2011) y Laos (2010). Esto podría deberse a que, si bien las participantes están pasando por un evento 
Tabla 5

Análisis de regresión como predictor de la CVRS mental

\begin{tabular}{lcccc}
\hline & \multicolumn{2}{c}{ Estadística por pasos } & \multicolumn{2}{c}{ Estadística por predictor } \\
\cline { 2 - 4 } Pasos y predictores & $\mathrm{R}^{2}$ & $\Delta \mathrm{R}^{2}$ & $\beta$ & $t$ \\
\hline Modelo con Estrategias & \multicolumn{4}{c}{ Ingreso de variables } \\
Paso 1 & 0.31 & $0.24^{* * *}$ & -0.30 & $-2.20^{* *}$ \\
$\quad$ Estrés traumático & 0.39 & $0.07^{* *}$ & -0.27 & -1.86 \\
$\quad$ Estrés percibido & & & & -1.12 \\
Paso 2 & 0.42 & 0.04 & -0.17 & -0.85 \\
$\quad$ Enfocar y liberar emociones & 0.43 & 0.01 & -0.12 & \\
$\quad$ Soporte social emocional & & & &
\end{tabular}

Modelo con Estilos

Paso 1

Estrés traumático

Estrés percibido

Paso 2

Estilo orientado a la emoción
Ingreso de variables

$\begin{array}{llll}0.30 & 0.30 * * * & -0.35 & -2.80^{* * *} \\ 0.37 & 0.08^{* *} & -0.33 & -2.73 * * *\end{array}$

0.37

0.42

$0.05^{*}$

$-0.23$

$-1.96^{*}$

$N=53,{ }^{*} p=.05,{ }^{* *} p<.05,{ }^{* * *} p<.01, \beta=$ beta estandarizado.

potencialmente estresante como es la enfermedad y su tratamiento (Koch, 2007; O’Connor et al., 2011), se encuentran ya bajo una condición de asistencia y cuidados, lo que les daría la sensación de contención y de manejo activo de la enfermedad. Quizás por ello, su estado actual difiere del imaginario colectivo que suele haber en torno a la enfermedad como sufrimiento, dolor y muerte (Die-Trill, 2003).

Agregado a ello, se encontró que el componente físico mantuvo un nivel promedio de CVRS, aunque se vio más afectado que el componente mental. Es decir, las participantes perciben mayor deterioro de sus funciones físicas en comparación con su función mental, coincidiendo así con anteriores investigaciones (Kershaw et al., 2004; Laos, 2010; Ransom et al., 2005, Torres, 2011).

La mayoría de dimensiones de la CVRS Física se encuentran preservadas, con excepción del rol físico, pues resultó ser la más deteriorada entre el grupo de participantes. Probablemente, este hallazgo refleje que las pacientes perciben como medianamente comprometido su nivel de funcionalidad en sus actividades cotidianas, lo cual podría articularse con el hecho de que la estrategia supresión de actividades competentes se correlacionó de manera inversa con el componente físico de la CVRS. Al mismo tiempo, indica que las pacientes, para ajustarse al proceso de enfermedad, deben postergar ciertas ocupaciones; lo que es coincidente con el hecho de que la mayoría de ellas se encuentra en un periodo activo de tratamiento (Schwartzmann, 2003).

En relación con los estilos de afrontamiento, se encuentra que hay similar frecuencia de uso del estilo orientado al problema y el estilo orientado a la emoción, siendo este último ligeramente más frecuente entre el grupo de participantes. Esto indicaría que, ante el padecimiento de un cáncer de mama, las mujeres se ven impulsadas a desplegar estrategias dirigidas a buscar soluciones a su enfermedad (tratamientos) y a su vez, cómo lidiar con las emociones que esto conlleva. 
Asimismo, se encontró un uso más reducido del afrontamiento evitativo, lo cual, según distintas investigaciones, resulta más adaptativo y de mejor impacto para la CVRS frente al cáncer (Constanzo et al., 2006; Van Laarhoven et al., 2011; Yang et al., 2008). Esto podría sustentar que en el nivel de la CVRS físico pero, sobre todo, mental se encuentren puntuaciones promedio y altas, pues las estrategias desplegadas favorecerían la reducción de emociones negativas como la ansiedad y facilitarían el acceso a estrategias más funcionales que permiten mayor bienestar ante una enfermedad grave.

A nivel de estrategias, la más usada fue el uso de la religión y reinterpretación positiva y crecimiento, lo que concuerda con los hallazgos de Gaviria et al. (2007) y Kershaw et al. (2004). En general, se observa que ciertas actitudes y prácticas religiosas o espirituales son factores importantes y positivos en el afrontamiento al cáncer. En ese sentido, el uso de la religión supone la posibilidad de adjudicarle un significado a la enfermedad y el tratamiento y, por lo tanto, facilitaría también la posibilidad de reinterpretar y re-focalizar el sentido de la enfermedad. La estrategia menos usada fue el uso de sustancias (alcohol o drogas), lo cual es esperable debido a la fase de tratamiento en la que se encuentran las participantes.

En cuanto a los componentes de la CVRS, se halló que para la CVRS Física, el estrés traumático, migración y tipo de tratamiento eran las variables que aportaban el 30\% de la variabilidad de dicho componente. En ese sentido, experimentar síntomas de estrés traumático estaría limitando, significativamente, el funcionamiento cotidiano de las mujeres evaluadas (Koch, 2007; O'Connor et al., 2011). Respecto al tipo de tratamiento, principalmente la quimioterapia, se vincula con una mejor CVRS Física. Esto implica que aun habiendo malestar físico como efecto secundario a este tratamiento, quizás dichos síntomas resultan ser menos limitantes que los producidos por el periodo postoperatorio.

Por otro lado, las pacientes migrantes a Lima obtienen menor CVRS Física, lo que podría explicarse porque estas mujeres dejan su ciudad para recibir tratamiento oncológico; usualmente, deben viajar solas o, en el mejor de los casos, acompañadas de un solo familiar. A ello, se suma el hecho de verse obligadas a acomodarse en contextos novedosos dentro de la ciudad capital y dentro del propio centro de salud, lo cual demanda conocer nuevos códigos, procedimientos, horarios y normas (Ferrer et al., 2014; Granel 2008; Hernández et al., 2009). Por eso, las mujeres migrantes podrían estar enfrentando un duelo múltiple que supone no solo elaborar la pérdida de la salud y sus capacidades físicas, sino también la pérdida o separación de la familia, los amigos, la cultura y, en ocasiones, la lengua, además de nuevas presiones económicas y financieras producto del abandono parcial o total del trabajo (Granel, 2008; MINSA, 2012).

En relación con el modelo explicativo del componente mental, este aporta un importante $43 \%$ de la variabilidad de la CVRS. El estrés traumático es, nuevamente, la variable que mayor variabilidad aportó, afectándola negativamente, incluso más que al componente físico; a dicho aspecto le sigue el estrés percibido, el cual coincide con investigaciones como las de Golden-Kreutz et al., (2005); finalmente, el uso de un estilo de afrontamiento centrado en la emoción también contribuye a explicar aspectos de la CVRS Mental.

La sintomatología traumática puede explicarse si las pacientes perciben al cáncer como un fenómeno amenazante del que no tienen control y que adquiere el carácter de impredecible, experimentándolo como una vivencia aterradora (Koch, 2007; O'Connor et al., 2011). En ese sentido, la vivencia estresante del cáncer puede pasar a convertirse en una experiencia traumática y, como tal, se experimentarán pensamientos catastróficos repetitivos u obsesivos que impiden el acceso a una buena calidad de sueño o descanso, se generarán estados de hiperactivación y, finalmente, interferirán en el bienestar de las pacientes y en su CVRS Mental.

Que el uso de un estilo de afrontamiento orientado a la emoción afectara negativamente la CVRS Mental podría deberse a la posibilidad de que para estas pacientes, reconocer y descargar sus estados emocionales negativos estaría ligado a una sensación 
de falta de control sobre su realidad, característica que incrementaría su sensación de indefensión, pérdida y depresión, lo cual impacta negativamente sobre la CVRS (Thompson \& Collins, 1995). En el Perú, se suelen usar términos que minimicen el impacto real de los sentimientos o pensamientos ya que la vivencia real de las emociones podría percibirse como agresiva; por lo que aquellas mujeres que hayan expresado abierta y directamente su sensación, pueden haber sufrido una reducción del soporte social como rechazo a la expresión de dichas emociones.

Con base en todos estos resultados, pareciera que la enfermedad en sí misma no produce un impacto devastador en la CVRS de las mujeres con cáncer de mama, sino que son algunas condiciones médicas, sociales, culturales, familiares y personales que lo acompañan, las que facilitan un adecuado ajuste a la enfermedad y, por lo tanto, repercuten en la CVRS de las pacientes.

El presente estudio sugiere la necesidad de identificar a los pacientes oncológicos como una población con necesidades médicas, psicológicas y sociales particulares. Estas personas necesitan ser estudiadas y comprendidas para poder intervenir adecuadamente en su proceso de recuperación o rehabilitación, poniendo más énfasis en la CVRS y no solo en la supervivencia de los pacientes. Asimismo, los hallazgos sugieren que se requiere poner especial atención en el tipo de estrés que las pacientes evaluadas experimentan, al tipo de tratamiento y a la condición de migración, por ser variables que suelen asociarse con una menor CVRS física.

En cuanto a las limitaciones del estudio, es necesario mencionar que el tamaño de muestra utilizado es relativamente pequeño y responde a las problemáticas sociales y económicas del país, así como a limitaciones de temporalidad en la medida que la investigación limitado por el tiempo. En ese sentido, se recomienda ampliar su cobertura a un número mayor de participantes.

Por otra parte, si bien el diseño transversal del presente estudio brinda luces sobre el funcionamiento actual de las variables examinadas, se podrían profundizar los hallazgos realizando una investigación longitudinal. Esto permitiría conocer la evolución o posibles cambios en el ajuste de las pacientes con cáncer de mama, lo cual, a su vez, ayudaría a delinear el tipo de intervención psicológica necesaria ante cada etapa de la enfermedad.

\section{Referencias}

Alonso, J., Prieto, L., \& Antó, J. (1995). La versión española del SF-36 Health Survey (Cuestionario de Salud SF-36): un instrumento para la medida de los resultados clínicos. Medicina Clínica, 104, 771-776.

Atef-vahid, M., Nasr-Esfahani, M., Saberi, M., NajiIsfahani, H., Reza, M., Yasavoli, M., \& Goushegir, S. (2011). Quality of life, religious attitude and cancer coping in a sample of Iranian patients with cancer. Journal of research in medical sciences, 16(7), 928-937.

Avis, N., Crawford, S., \& Manuel, J. (2005). Quality of life among younger women with breast cancer. Journal of Clinical Oncology, 23(15), 3322-3330. doi: 10.1200/JCO.2005.05.130

Blake, D. D., Weathers, F. W., Nagy, L. M., Kaloupek, D. G., Gusman, F. D., Charney, D. S., \& Keane, T. M. (1995). The development of a clinicianadministered PTSD scale. Journal of Traumatic Stress, 8, 75-90.

Cassaretto, M., \& Chau, C. (2016). Afrontamiento al estrés: adaptación del cuestionario COPE en universitarios de Lima. Revista Iberoamericana de Diagnóstico y Evaluación e Avaliação Psicológica, 2(42), 95-109. doi: 10.21865/RIDEP42_95

Carver, C., Kumari, J., \& Scheier, M. (1989). Assessing coping strategies: A theoretically based approach. Journal of Personality and Social Psychology, 56(2), 267283. doi: 10.1037/0022-3514.56.2.267

Castellá, J. (2003). Estudios actuales sobre aculturación en latinos: revisión y nuevas perspectivas. Revista Interamericana de Psicología, 37(2), 341-364.

Cohen, S., Kamarck T., \& Mermelstein, R. (1983). A global measure of perceived stress. Journal of bealth and social behavior, 24(4), 385-396. 
Costa-Requena, G., Rodríguez, A., \& Fernández-Ortega, P. (2012). Longitudinal assessment of distress and quality of life in the early stages of breast cancer treatment. Scandinavian Journal of Caring Sciences, 27(1), 77-83. doi: 10.1111/j.1471-6712.2012.01003.x

Costanzo, E., Lutgendorf, S., Mattes, M., Trehan, S., Robinson, C., Tewfik, F., \& Roman, S. (2007). Adjusting to life after treatment: distress and quality of life following treatment for breast cancer. British Journal of Cancer, 97(12), 1625-1631. doi: 10.1038/ sj.bjc.6604091

Costanzo, E., Lutgendorf, S., Rothrock, N. \& Anderson, B. (2006). Coping and quality of life among women extensively treated for gynecologic cancer. Psychooncology, 15(2), 132-142. doi: 10.1002/pon.930

Da Silva, G., \& Dos Santos, M. (2010). Stressors in breast cancer post-treatment: a qualitative approach. Revista Latino-americana Enfermagem, 18(4), 688-695. doi: 10.1590/S0104-11692010000400005

Die Trill, M. (2003). Influencia de la cultura en la experiencia del cáncer. Psicooncología, 0(1), 39-48.

Ferrer, R., Palacio, J., Hoyos, O., \& Madariaga, C. (2014). Proceso de aculturación y adaptación del inmigrante: características individuales y redes sociales. Psicología desde el Caribe, 31(3), 557-576. doi: 10.14482/psdc.31.3.4766

Filazoglu, G., \& Griva, K. (2008). Coping and social support and health related quality of life in women with breast cancer in Turkey. Psychology, health and medicine, 13(5), 559-573. doi: 10.1080/13548500701767353

Galloway, S., Baker, M., Giglio, P., Chin, S., Madan, A., Malcolm, R., Serber, E., Wedin, S., Balliet, W., \& Borckardt, J. (2012). Depression and Anxiety Symptoms Relate to Distinct Components of Pain Experience among Patients with Breast Cancer. Pain research and treatment, 2012(2012), 1-4. doi: 10.1155/2012/851276

Gargurevich, R. (2006). Posttraumatic Stress Disorder and Disasters in Peru: The Role of Personality and Social Support. (Tesis de doctorado). Universidad Católica de Leuven, Bélgica.

Gaviria, A., Vinaccia, S., Riveros, M., \& Quinceno, J. (2007). Calidad de vida relacionada con la salud, afrontamiento del estrés y emociones negativas en pacientes con cáncer en tratamiento quimioterapéutico. Psicología desde el Caribe, (20), 50-75.

Gil, A., Wagner, E., \& Vega, W. (2000). Acculturation, familism and alcohol use among Latino adolescent males: Longitudinal relations. Journal of Community Psychology, 28(4), 443-458. doi: 10.1002/1520-6629(200007)28:4<443::AIDJCOP6>3.0.CO;2-A

Golden-Kreutz, D., Thornton, L., Wells-DiGregorio, S., Frierson, G., Jim, H., Carpenter, K., Shleby, R., \& Andersen, B. (2005). Traumatic stress, perceived global stress and life events: Prospectively predicting quality of life in breast cancer patients. Health Psychology, 24(3), 288-296. doi: 10.1037/02786133.24.3.288

Granel, L. (2008). El desarraigo y duelo migratorio en la experiencia de la migración. (Tesis inédita de maestría). PUCP, Lima.

Gurevich, M., Devins, G. \& Rodin, G. (2002). Stress response syndromes and cancer: conceptual and assessment issues. Psychosomatics, 43(4), 259-281. doi: 10.1176/appi.psy.43.4.259

Hernández, R., Fernández, C., \& Baptista P. (2010). Metodología de Investigación. 5ta ed. Ciudad de México, México: McGraw - Hill.

Hernández, M., Lorenzo, R., \& Nacif, L. (2009). Impacto emocional: cáncer infantil - inmigración. Psicooncología, 6(2-3), 445-457.

Holmes, T. \& Rahe, R. (1976). The social readjustment rating scale. J. Psychoson. Res., 11, 213-218

Horowitz, M., Wilner, N., \& Álvarez, W. (1979). Impact of events scale: A measure of subjective stress. Psychosomatic Medicine, 41(3), 209-218.

Instituto Nacional de Enfermedades Neoplásicas. (2016). Indicadores de gestión hospitalaria. Recuperado de: http://www.inen.sld.pe/portal/documentos/pdf/ 
estadistica/estadisticas/29082016_Julio_2016.pdf

Karakajun - Celik, O., Ilknur, G., Sahin, S., Orcin, E., Alanyali, H., \& Kinay, M. (2010). Depression and anxiety levels in women under follow-up for breast cancer: relationship to coping with cancer and quality of life. Medicina Oncológica, 27(1), 108-113. doi: 10.1007/s12032-009-9181-4

Kershaw, T., Northouse, L. Kritpracha, C., Schafenacker, A., \& Mood, D. (2004). Coping strategies and quality of life in women with advanced breast cancer and their family caregivers. Psychology and Health, 19(2), 139-155.

Kline, R. (2010). Principles and practice of structural equation modeling (3era ed.). New York; Guilford Press.

Koch, M. (2007). Prevalence of acute and posttraumatic stress disorder and comorbid mental disorders in breast cancer patients during primary cancer care: a prospective study. Psychooncology, 16(3), 181-188. doi: 10.1002/pon.1057

Koopman, C., Butler, L., Clessen, C., Giese-Davis, J., Morrow, G., Westendorf, J., Banerjee, T., \& Spiegel, D. (2002). Traumatic stress symptoms among women with recently diagnosed primary breast cancer. Journal of Trauma Stress, 15(4), 277-287. doi: 10.1023/A:1016295610660

Laos, K. (2010). Calidad de vida y religiosidad en pacientes con cáncer de mama. (Tesis inédita de licenciatura). PUCP, Lima.

Lazarus, R., \& Folkman, S. (1986). Estrés y procesos cognitivos. Barcelona, España: Martínez Roca.

Lazarus, R., \& Lazarus, B. (2000). Pasión y razón. La comprensión de nuestras emociones. España: Ediciones Paidós Ibérica.

Ministerio de Salud del Perú. (2012). Plan Esperanza. Plan Nacional para la atención integral del cáncer y el mejoramiento del acceso a servicios oncológicos en el Perú. Recuperado de: http://www.minsa.gob.pe/ portada/especiales/2012/esperanza/

New Jersey Department of Health. (2013). Office of Minority and Multicultural Health. Recuperado de: http://www.nj.gov/health/omh/background_ sp.shtml

O'Connor, M., Christensen, S., Jensen, A., Moller, S., \& Zachariae, R. (2011). How traumatic is breast cancer? Post-traumatic stress symptoms (PTSS) and risk factors for severe PTSS at 3 and 15 months after surgery in a nationwide cohort of Danish women treated for primary breast cancer. British Journal of Cancer, 104(3), 419-426. doi: 10.1038/sj.bjc.6606073

Organización Mundial de la Salud OMS. (1995). The World Health Organization Quality of life assessment (WHOQOL). Position Paper from the World Health Organization. Social Science \& Medicine, 41(10), 1403-1409.

Organización Mundial de la Salud OMS. (2012). Cáncer de mama: prevención y control. Recuperado de: http://www. who.int/topics/cancer/breastcancer/es/index1.html

Ransom, S., Jacobsen, P., Schmidt, J., \& Andrykowski, M. (2005). Relationship of problem-focused coping strategies to changes in quality of life following treatment for early stage breast cancer. Journal of Pain Symptom Manage, 30(3), 243-253. doi: 10.1016/j. jpainsymman.2005.03.013

Reich, M., \& Remor, E. (2014). Psychological Variables Associated with Health-Related Quality-of-Life in Uruguayan Women Surgically Intervened for Breast Cancer. Psychology, Community \& Health, 3(3), 172188. doi: $10.5964 /$ pch.v3i3.98

Remor, E. (2006). Psychometric Properties of a European Spanish Version of the Perceived Stress Scale (PSS). The Spanish Journal of Psychology, 9(1), 86-93.

Remor, E., \& Carrobles, J. (2001). Versión española de la Escala de Estrés Percibido (PSS-14): estudio psicométrico en una muestra VIH+. Ansiedad y Estrés, 7(2-3), 195-201.

Rincón, M., Pérez, M., Borda, M., \& Martín, A. (2010). Diferencias psicológicas en pacientes con cáncer de mama según el tipo de cirugía mamaria. Cirugía plástica iberolatinoamericana, 36(4), 359-368.

Salazar, F., \& Bernabé, E. (2012). The Spanish SF-36 
in Peru: Factor Structure, Construct Validity, and Internal Consistency. Asia Pac Journal of Public Health, 27(2), 2372-2380. doi: 10.1177/1010539511432879

Schwartzmann, L. (2003). Calidad de vida relacionada con la salud: aspectos conceptuales. Ciencia y enfermería, 9(2), 9-21. doi: 10.4067/S071795532003000200002

Shelby, R., Golden-Kreutz, D., \& Andersen, B. (2008). PTSD diagnoses, subsyndromal symptoms and comorbidities contribute to impairments for breast cancer survivors. Journal of Trauma Stress, 21 (2), 165172. doi: $10.1002 /$ jts.20316

Shishehgar, S., Gholizadeh, L., DiGiacomo, M., \& Davidson, P. (2015). The impact of migration on the health status of Iranians: an integrative literature review. International Health and Human Rights, 15(1), 2-11. doi: 10.1186/s12914-015-0058-7

Stafford, L., Judd, F., Gibson, P., Komiti, A., Mann, G., \& Quinn, M. (2013). Screening for depression and anxiety in women with breast and gynecologic cancer: course and prevalence of morbidity over 12 months. Psychooncology, 22(5), 2071-2078. doi: $10.1002 /$ pon.3253

Sundin, E., \& Horowitz, M. (2002). Impact of event scale: Psychometric properties. British Journal of Psychiatry, 180(3), 205-209. doi: 10.1192/ bjp.180.3.205

Tarakeshwar, N., Vanderwerker, L., Paulk, E., Pearce, M., Kasl, S., \& Prigerson, H. (2006). Religious coping is associated with the quality of life of patients with advanced cancer. Journal of Palliative Medicine, 9(3), 646-657. doi: 10.1089/jpm.2006.9.646

Thompson, S., \& Collins, M. (1995). Applications of perceived control to cancer: An overview of theory and measurement. En Curbow, B. \& Somerfield, M. (Ed.), Psychosocial Resource Variables in Cancer Studies: Conceptual and Measurement Issues. New York: Haworth Press.

Torres, J. (2011). Relación entre calidad de vida relacionada con la saludy soporte social en pacientes con cáncer de mama. (Tesis inédita de licenciatura). PUCP, Lima.

Van Laarhoven, H., Schilderman, J., Bleijenberg, G., Donders, R., Vissers, K., Verhagen, C., \& Prins, J. (2011). Coping, Quality of life, depression and hopelessness in cancer patients in a curative and palliative end-of-life setting. Cancer Nursing, 34(4), 302-314. doi: 10.1097/NCC.0b013e3181f9a040

Vilaglut, G., Ferrera, M., Rajmilb, L., Rebolloc, P., Permanyer-Miraldad, G., Quintanae, J., Santeda, R., Valderasa, J., Riberad, A., Domingo-Salvanya, A. \& Jordi Alonso (2005). El Cuestionario de Salud SF36 español: una década de experiencia y nuevos desarrollos. Gaceta Sanitaria, 19(2), 135-150

Ware, J. (1998). The factor structure of SF-36 Health Survey in 10 countries: Results from the international quality of life assessment (IQOLA) project. Journal of Epidemiology, 51(11), 1159-1165.

Ware, J. \& Sherbourne, C. (1992). The MOS 36-item short form health survey (SF-36) I. Medical Care, 30(1), 473-483.

Yamada, G. (2010). Migración interna en el Perú. Lima, Perú: Universidad del Pacífico.

Yang, H., Brothers, B., \& Andersen, B. (2008). Stress and Quality of Life in Breast Cancer Recurrence: Moderation or Mediation of Coping? Annals of Behavioral Medicine, 35(2), 188-197. doi: 10.1007/ s12160-008-9016-0

Recibido: 27 de junio de 2016

Aceptado: 19 de octubre de 2016 